\title{
First-Line Treatment with Bevacizumab and Platinum Doublet Combination in Non-Squamous Non-Small Cell Lung Cancer: A Retrospective Cohort Study in US Oncology Community Practices
}

\author{
Orsolya E. Lunacsek ${ }^{1}$ Arliene Ravelo ${ }^{2}$ - Anna D. Coutinho ${ }^{1}$ - Sebastien J. Hazard ${ }^{2}$ • \\ Mark R. Green ${ }^{1} \cdot$ Joanne Willey $^{1} \cdot$ Michael Eaddy $^{1} \cdot$ Hans-Peter Goertz $^{2}$
}

Published online: 31 August 2016

(C) The Author(s) 2016. This article is published with open access at Springerlink.com

\begin{abstract}
Background Real-world evidence is lacking on the impact of bevacizumab added to carboplatin/paclitaxel (Bev + $\mathrm{CP})$ therapy versus $\mathrm{CP}$ alone for patients with non-squamous non-small cell lung cancer (NS-NSCLC), particularly in those excluded from clinical trials.

Methods This is a retrospective electronic medical record analysis of patients who received first-line therapy with Bev + CP or CP between 1 October 2006 and 30 June 2013. We identified four subsets: elderly patients $(\geq 65$ years), patients with brain/central nervous system (CNS) metastases, patients with Eastern Cooperative Oncology Group performance status (ECOG PS) $\geq 2$, and patients receiving anticoagulation. We used descriptive statistics to describe patient characteristics and treatment patterns and evaluated progression-free survival (PFS) and overall survival (OS) using survival analyses.

Results The study included 431 patients (Bev + CP: 231; CP: 200). The Bev + CP cohort was more likely to receive four or more cycles of induction therapy (72 vs. $50 \%$ ) and was more likely to receive maintenance therapy ( 45 vs. 21 $\%)$ than patients receiving $\mathrm{CP}$. In the overall population, median PFS and OS were significantly longer in the Bev + $\mathrm{CP}$ cohort than in the $\mathrm{CP}$ cohort: 6.7 vs. 5.1 months (hazard ratio [HR] 0.74; $95 \%$ confidence interval [CI] 0.59-0.92;
\end{abstract}

Orsolya E. Lunacsek

orsolya.lunacsek@xcenda.com

1 Xcenda LLC, 4114 Woodlands Parkway, Suite 500, Palm Harbor, FL, USA

2 Genentech, Inc., 1 DNA Way, San Francisco, CA 94080 USA $p=0.008)$ and 11.9 vs. 9.0 months (HR 0.57; $95 \% \mathrm{CI}$ $0.44-0.73 ; p<0.001)$, respectively. Treatment with Bev + $\mathrm{CP}$ in patients aged $\geq 65$ years and in those with brain/CNS metastases was also associated with a significant risk reduction in PFS (35 and $51 \%$, respectively; $p<0.05$ for both) and OS (46 and $62 \%$, respectively; $p<0.05$ for both) compared with $\mathrm{CP}$ alone.

Conclusion Bev $+\mathrm{CP}$ is associated with a significant improvement in PFS and OS in patients with NS-NSCLC and in subsets with brain/CNS metastases and those aged $\geq 65$ years.

\section{Key Points}

Despite that the current guidelines for the treatment of non-small cell lung cancer (NSCLC) endorse bevacizumab added to the chemotherapy backbone of carboplatin and paclitaxel, there is a lack of published real-world evidence documenting the use of this combination in subpopulations either excluded or under-represented in clinical trials.

Our study suggested that bevacizumab, when added to carboplatin and paclitaxel in a real-world setting, is associated with a survival advantage; this advantage is also seen in patients with brain/central nervous system metastases and in patients aged $\geq 65$ years.

Understanding what populations stand to benefit in a real-world setting from the use of bevacizumab may influence future guideline development and provide direction regarding outcomes of interest in future trials in NSCLC. 


\section{Introduction}

Non-small cell lung cancer (NSCLC) is the leading cause of cancer-related deaths in the USA, accounting for $>25 \%$ of all cancer-related deaths in both men and women [1]. At diagnosis, $70 \%$ of patients have advanced or metastatic disease, and systemic chemotherapy is the main treatment modality employed to prolong survival [2]. Historically, platinum-based doublets have been the backbone of treatment for advanced or metastatic NSCLC, with carboplatin and paclitaxel having a toxicity advantage over other platinum-based comparators [3, 4]. More recently, histology has been elucidated as a major driver in determining sensitivity to specific chemotherapy combinations $[5,6]$.

A study by the Eastern Cooperative Oncology Group (ECOG), study ECOG 4599, revealed that the addition of bevacizumab to the carboplatin/paclitaxel $(\mathrm{Bev}+\mathrm{CP})$ backbone with continued bevacizumab maintenance has a survival advantage of approximately 2 months versus carboplatin/paclitaxel (CP) alone in non-squamous NSCLC (NS-NSCLC); this advantage is even more pronounced in patients with adenocarcinoma histology [5, 7]. A second European trial, AVAiL, found that the addition of bevacizumab at two different doses to another platinum backbone, gemcitabine and cisplatin, significantly prolonged progression-free survival (PFS) but was not powered to detect an overall survival (OS) advantage [8]. While these studies have placed bevacizumab in combination with a platinum-based regimen among the recommended first-line therapies for NS-NSCLC [9], the exclusion criteria employed by these trials limit the evidence regarding the efficacy of bevacizumab in a real-world setting. Specifically, patients with brain or central nervous system (CNS) metastases, poor performance status (PS; i.e., ECOG PS $\geq 2$ ), and those receiving therapeutic anticoagulation were excluded. For the population using bevacizumab with anticoagulants, no increased risk has been observed in those with non-squamous histology based on the retrospective analysis of clinical trial data [10, 11]. However, real-world analyses evaluating whether the use of anticoagulants in patients with NS-NSCLC reduces the effectiveness of bevacizumab are lacking. Therefore, this subset was included as a subpopulation of interest in the current study.

Further, in the ECOG 4599 trial, although $>40 \%$ of patients were aged $\geq 65$ years, subset analysis revealed this population had more limited benefit from the addition of bevacizumab than their younger counterparts: hazard ratio (HR) for death: 0.89 (95\% confidence interval [CI] $0.70-1.14$ ) vs. 0.71 (95\% CI 0.58-0.88), respectively [7]. More recent literature has suggested that the addition of bevacizumab to chemotherapy is beneficial for patients aged up to 75 years, but the benefit in those aged $\geq 75$ years is unconfirmed [12-14].

With the increasing number of therapeutic options, considerable effort has been directed toward identifying optimal therapies for specific segments of the NS-NSCLC patient population given the paucity of evidence in this area. The goal of this retrospective cohort study was to assess real-world utilization and outcomes of patients with advanced-stage NS-NSCLC receiving first-line Bev + CP or $\mathrm{CP}$ without bevacizumab. Within the full study population, results were also stratified to address information gaps in the literature for four subpopulations: elderly patients aged $\geq 65$ years, patients with brain or CNS metastases, patients with an ECOG PS $\geq 2$, and patients with recent or ongoing anticoagulant therapy.

\section{Methods}

\subsection{Data Source}

For this study, we used data from the International Oncology Network (ION) electronic medical record (EMR) database between April 2006 and July 2013. ION is a geographically diverse physician services network, the membership of which represents over half of the private practice oncologists in the USA. The ION EMR database includes both standardized EMR tables and electronic patient progress notes. Data for this study were obtained from the standardized tables; a comprehensive manual chart review of progress notes supplemented information from the tables. Data collected included patient characteristics regarding diagnosis, age, sex, ECOG PS, smoking status, comorbidities, anticoagulant use, vitals (height, weight, blood pressure, etc.) and disease/treatment characteristics such as extent of disease, treatment plan schedule, treatment response, and disease progression. Vital status, including date of death, was supplemented with data from the Social Security Death Master File.

\subsection{Study Design and Population}

This was a retrospective cohort analysis of patients with advanced or metastatic NS-NSCLC who received first-line induction therapy with $\mathrm{Bev}+\mathrm{CP}$ or $\mathrm{CP}$ between 1 October 2006 and 30 June 2013. The start date of first-line induction therapy was defined as the study index. A 6-month period before the index was used as the baseline period to characterize the study population. Patients were then followed from index until the earliest of last recorded visit date, date of progression or death, or end of study period (31 July 2013). All patients were required to have a minimum of 1 
month of follow-up after index, which excluded patients who progressed or died within the first 30 days following index and ensured receipt of at least one cycle of first-line induction therapy.

Patients were required to be aged $\geq 18$ years, have a confirmed diagnosis of stage IIIB or stage IV NS-NSCLC, have confirmed non-squamous histology, and have received first-line induction therapy within 180 days of diagnosis of advanced/metastatic disease. Patients were excluded if they had evidence of other primary malignancies, had incomplete progress notes, or had received other chemotherapy or investigational agents during first-line induction therapy. Additionally, patients with concurrent radiotherapy during induction therapy, indicating radiosensitizing chemotherapy, were also excluded.

The four subpopulations of interest were defined as follows: (1) elderly patients aged $\geq 65$ years at index; (2) patients with brain or CNS metastases at index or any time during the 6-month baseline period, defined as the presence of a diagnosis code for distant metastases in the brain (International Classification of Diseases, Ninth Revision, Clinical Modification [ICD-9-CM] codes 198.3, 198.4) in the standardized EMR tables or the presence of iterations of the following terms in the progress notes: 'brain metastases,' 'brain mets,' 'CNS metastases,' or equivalent terms; (3) patients with an ECOG PS score of $\geq 2$; ECOG score closest to index was captured and only scores within 3 months before or after index were captured; and (4) patients with recent or ongoing anticoagulant therapy, defined as the presence of oral or parenteral anticoagulants in the progress notes at index or within 3 months before or 1 month after index.

\subsection{Outcome Measures}

The primary outcome measures were PFS and OS. PFS was defined as the time from index to progression, which was assessed within the progress notes as mention of disease progression or mixed response, initiation of hospice, or death. Patients who were lost to follow-up or who reached the end of the study period without evidence of progression or death event were censored for the PFS analysis. OS was defined as the time from first-line therapy initiation to date of death; patients were only censored if they were lost to follow-up or reached the end of the observation period ( 31 July 2013) for the OS analysis.

To characterize treatment patterns, we evaluated the total number of cycles of induction therapy, the proportion of patients treated according to label for induction therapy defined as having four or more cycles, the proportion of patients treated with maintenance therapy after induction, and the proportion of patients treated with second-line therapy. Maintenance therapy was defined as receipt of therapy after end of induction therapy but before progression. Second-line therapy was defined as receipt of therapy after disease progression. Additionally, the discontinuation rate of both induction and maintenance therapy was also reported together with reasons for discontinuation and reasons for not initiating maintenance therapy.

\subsection{Statistical Analysis}

We used descriptive statistics (frequencies, proportions, means, medians, standard deviations) to describe patient demographic/clinical characteristics and treatment patterns by treatment cohort and inferential statistics to assess differences at baseline between cohorts using Chi-squared tests for categorical variables and $t$ tests for continuous variables. We used univariate Kaplan-Meier and multivariate Cox proportional hazards $(\mathrm{PH})$ regression analyses to estimate and compare OS and PFS by treatment cohort. Results of the Kaplan-Meier analyses included median survival and associated $p$ values based on a log-rank test. Results of the Cox models were summarized by highlighting HRs and associated $95 \%$ CIs for the independent variables. The Cox PH models adjusted for the following covariates: age at therapy initiation, sex, geographic region, payer, year of therapy initiation (before 2009 [reference] or later), smoking status, ECOG PS score, Charlson comorbidity index score in the 6 months prior to therapy initiation, presence of brain or CNS metastasis, and presence of bone metastasis. We selected the year of therapy initiation as a covariate because a shift in treatment paradigm toward more bevacizumab use started in 2009.

\section{Results}

We identified 4424 patients with NS-NSCLC who initiated treatment with $\mathrm{Bev}+\mathrm{CP}$ or $\mathrm{CP}$ between 1 October 2006 and 30 June 2013 (Fig. 1). Of these, 431 (Bev + CP: $n=$ 231; CP: $n=200$ ) met study inclusion criteria. The majority of patients were excluded because of a lack of evidence for metastatic or advanced disease $(62.4 \%)$ and an inability to confirm non-squamous histology (18.3\%) (Fig. 1). Within the pre-specified subsets, 231 patients were aged $\geq 65$ years, 96 had brain/CNS metastases, 57 had an ECOG PS $\geq 2$, and 58 were receiving anticoagulation therapy at initiation of first-line induction therapy with Bev $+\mathrm{CP}$ or $\mathrm{CP}$.

Table 1 summarizes the patient characteristics for the overall population and by treatment with or without bevacizumab. The sample was almost evenly split at the cutoff age of 65 years, with $54 \%$ of the sample aged $\geq 65$ years. Patients aged $\geq 70$ years accounted for almost onethird of the patients, with $16 \%$ aged $\geq 75$ years. In general, 


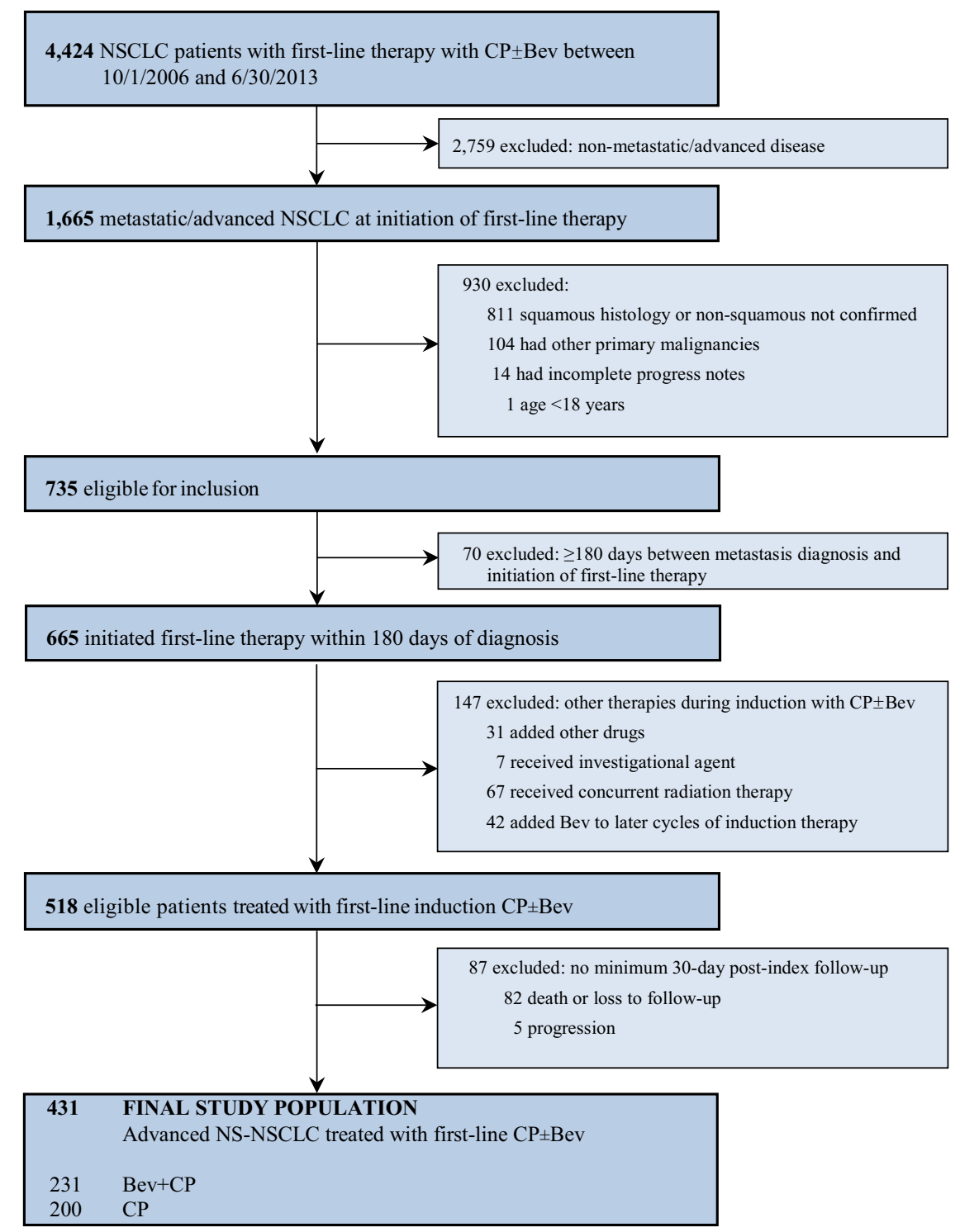

\section{SUBPOPULATIONS}

\begin{tabular}{|rl|}
\hline 231 & Elderly \\
137 & $\mathrm{Bev}+\mathrm{CP}$ \\
94 & $\mathrm{CP}$ \\
\hline
\end{tabular}

\begin{tabular}{|ll|}
96 & Brain/CNS metastases \\
25 & $\mathrm{Bev}+\mathrm{CP}$ \\
71 & $\mathrm{CP}$ \\
\hline
\end{tabular}

\begin{tabular}{|ll|}
\hline 57 & ECOG PS $\geq 2$ \\
28 & $\mathrm{Bev}+\mathrm{CP}$ \\
29 & $\mathrm{CP}$ \\
\hline
\end{tabular}

\begin{tabular}{|ll|}
\hline 58 & Anticoagulant use \\
24 & Bev $+\mathrm{CP}$ \\
34 & $\mathrm{CP}$ \\
\hline
\end{tabular}

Fig. 1 Final study population and subpopulations. Bev bevacizumab, $C N S$ central nervous system, $C P$ carboplatin + paclitaxel, ECOG Eastern Cooperative Oncology Group, NSCLC non-small cell lung cancer, NS-NSCLC non-squamous non-small cell lung cancer, $P S$ performance status

patients receiving $\mathrm{Bev}+\mathrm{CP}$ were older than patients receiving CP (66 vs. 64 years; $p=0.045$ ); accordingly, a higher proportion of patients were aged 65-74 years, but a similar proportion were aged $\geq 75$ years. The majority of the entire study population was male $(56 \%)$ with no difference between cohorts. ECOG PS was not significantly different between cohorts, with $64 \%$ of the Bev $+\mathrm{CP}$ cohort and $56 \%$ of the $\mathrm{CP}$ cohort classified as ECOG 0 or 1 and $12.1 \%$ of the $\mathrm{Bev}+\mathrm{CP}$ group and $14.5 \%$ of the $\mathrm{CP}$ group classified as ECOG $\geq 2$. The ECOG PS score was unknown in $24 \%$ of the Bev + CP group and in $30 \%$ of the $\mathrm{CP}$ group. The $\mathrm{Bev}+\mathrm{CP}$ cohort was significantly more likely than the $\mathrm{CP}$ cohort to have bone metastases (55 vs. $38 \% ; p<0.001)$ and was less likely than the $\mathrm{CP}$ cohort to have brain/CNS metastases (11 vs. $36 \% ; p<0.001)$. The comorbid burden, as measured by the Charlson 
Table 1 Demographic and clinical characteristics at baseline

\begin{tabular}{|c|c|c|c|c|}
\hline & All patients $(N=431)$ & $\mathrm{BEV}+\mathrm{CP}(n=231)$ & $\mathrm{CP}(n=200)$ & $p$ value $^{\mathrm{a}}$ \\
\hline Age in years & $64.6 \pm 9.8$ & $65.5 \pm 67.0$ & $63.6 \pm 63.0$ & 0.045 \\
\hline \multicolumn{5}{|l|}{ Age group, years } \\
\hline $18-64$ & $200(46.4)$ & $94(40.7)$ & $106(53.0)$ & \\
\hline $65-69$ & $86(20.0)$ & $54(23.4)$ & $32(16.0)$ & \\
\hline $70-74$ & $75(17.4)$ & $45(19.5)$ & $30(15.0)$ & \\
\hline$\geq 75$ & $70(16.2)$ & $38(16.5)$ & $32(16.0)$ & \\
\hline Male & $239(55.5)$ & $126(54.5)$ & $113(56.5)$ & 0.684 \\
\hline Race/ethnicity & & & & 0.010 \\
\hline Caucasian & $236(54.8)$ & $127(55.0)$ & $109(54.5)$ & \\
\hline African American & $32(7.4)$ & $11(4.8)$ & $21(10.5)$ & \\
\hline Asian & $2(0.5)$ & $2(0.9)$ & $0(0.0)$ & \\
\hline Hispanic & $2(0.5)$ & $0(0.0)$ & $2(1.0)$ & \\
\hline Other & $4(0.9)$ & $0(0.0)$ & $4(2.0)$ & \\
\hline Missing & $155(36.0)$ & $91(39.4)$ & $64(32.0)$ & \\
\hline Patient insurance & & & & 0.002 \\
\hline Medicaid & $22(5.1)$ & $6(2.6)$ & $16(8.0)$ & \\
\hline Medicare & $186(43.2)$ & $114(49.4)$ & $72(36.0)$ & \\
\hline Other & $5(1.2)$ & $0(0.0)$ & $5(2.5)$ & \\
\hline Private & $168(39.0)$ & $85(36.8)$ & $83(41.5)$ & \\
\hline Missing & $50(11.6)$ & $26(11.3)$ & $24(12.0)$ & \\
\hline Geographic region of practice & & & & 0.881 \\
\hline Midwest & $91(21.1)$ & $47(20.3)$ & $44(22.0)$ & \\
\hline Northeast & $30(7.0)$ & $15(6.5)$ & $15(7.5)$ & \\
\hline South & $283(65.7)$ & $153(66.2)$ & $130(65.0)$ & \\
\hline West & $27(6.3)$ & $16(6.9)$ & $11(5.5)$ & \\
\hline \multicolumn{5}{|l|}{ Location of metastases } \\
\hline Adrenal glands & $77(17.9)$ & $37(16.0)$ & $40(20.0)$ & 0.282 \\
\hline Bone & $201(46.6)$ & $126(54.5)$ & $75(37.5)$ & $<0.001$ \\
\hline Brain & $96(22.3)$ & $25(10.8)$ & $71(35.5)$ & $<0.001$ \\
\hline Contralateral lung & $64(14.8)$ & $37(16.0)$ & $27(13.5)$ & 0.464 \\
\hline Liver & $81(18.8)$ & $49(21.2)$ & $32(16.0)$ & 0.167 \\
\hline Lymph nodes & $232(53.8)$ & $116(50.2)$ & $116(58.0)$ & 0.106 \\
\hline Pericardium & $21(4.9)$ & $8(3.5)$ & $13(6.5)$ & 0.144 \\
\hline Pleural surface & $90(20.9)$ & $54(23.4)$ & $36(18.0)$ & 0.171 \\
\hline Other & $19(4.4)$ & $5(2.2)$ & $14(7.0)$ & 0.015 \\
\hline ECOG PS & & & & 0.348 \\
\hline 0 & $99(23.0)$ & $52(22.5)$ & $47(23.5)$ & \\
\hline 1 & 159 (36.9) & $95(41.1)$ & $64(32.0)$ & \\
\hline 2 & $50(11.6)$ & $24(10.4)$ & $26(13.0)$ & \\
\hline$\geq 3$ & 7 (1.6) & $4(1.7)$ & $3(1.5)$ & \\
\hline Unknown & $116(26.9)$ & $56(24.2)$ & $60(30.0)$ & \\
\hline Smoking status & & & & 0.327 \\
\hline Current smoker & $150(34.8)$ & $80(34.6)$ & $70(35.0)$ & \\
\hline Prior smoker (quit) & $214(49.7)$ & $109(47.2)$ & $105(52.5)$ & \\
\hline Never smoked & $47(10.9)$ & $28(12.1)$ & $19(9.5)$ & \\
\hline Unknown & $20(4.6)$ & $14(6.1)$ & $6(3.0)$ & \\
\hline
\end{tabular}


Table 1 continued

\begin{tabular}{|c|c|c|c|c|}
\hline & All patients $(N=431)$ & $\mathrm{BEV}+\mathrm{CP}(n=231)$ & $\mathrm{CP}(n=200)$ & $p$ value $^{\mathrm{a}}$ \\
\hline BMI & & & & 0.344 \\
\hline Underweight & $22(5.1)$ & $8(3.5)$ & $14(7.0)$ & \\
\hline Normal & $168(39.0)$ & $88(38.1)$ & $80(40.0)$ & \\
\hline Overweight & $144(33.4)$ & $80(34.6)$ & $64(32.0)$ & \\
\hline Obese & $97(22.5)$ & $55(23.8)$ & $42(21.0)$ & \\
\hline Charlson comorbidity index score & $6.7 \pm 0.9$ & $6.7 \pm 0.8$ & $6.7 \pm 1.1$ & 0.760 \\
\hline \multicolumn{5}{|l|}{ Comorbid conditions } \\
\hline Diabetes without end-organ damage & $65(15.1)$ & $34(14.7)$ & $31(15.5)$ & 0.821 \\
\hline Hypertension & $203(47.1)$ & $105(45.5)$ & $98(49.0)$ & 0.462 \\
\hline COPD & $124(28.8)$ & $70(30.3)$ & $54(27.0)$ & 0.450 \\
\hline Congestive heart failure & $9(2.1)$ & $5(2.2)$ & $4(2.0)$ & 1.000 \\
\hline Renal insufficiency/failure & $10(2.3)$ & $3(1.3)$ & $7(3.5)$ & 0.130 \\
\hline Anemia & $10(2.3)$ & $1(0.4)$ & $9(4.5)$ & 0.007 \\
\hline Thrombocytopenia & $5(1.2)$ & $3(1.3)$ & $2(1.0)$ & 1.000 \\
\hline History of myocardial infarction & $22(5.1)$ & $13(5.6)$ & $9(4.5)$ & 0.596 \\
\hline History of stroke/TIA & $10(2.3)$ & $7(3.0)$ & $3(1.5)$ & 0.351 \\
\hline History of bleeding events, serious hemorrhage, recent hemoptysis & $29(6.7)$ & $9(3.9)$ & $20(10.0)$ & 0.012 \\
\hline Cerebrovascular, valvular disease & $7(1.6)$ & $3(1.3)$ & $4(2.0)$ & 0.709 \\
\hline Connective tissue disease, rheumatologic disorders & $10(2.3)$ & $6(2.6)$ & $4(2.0)$ & 0.758 \\
\hline Peripheral vascular disease & $18(4.2)$ & $6(2.6)$ & $12(6.0)$ & 0.078 \\
\hline Ulcer disease & $5(1.2)$ & $1(0.4)$ & $4(2.0)$ & 0.188 \\
\hline Weight loss & 89 (20.6) & $33(14.3)$ & $56(28.0)$ & $<0.001$ \\
\hline Cardiovascular disease & $76(17.6)$ & $45(19.5)$ & $31(15.5)$ & 0.280 \\
\hline Venous thromboembolism & $28(6.5)$ & $8(3.5)$ & $20(10.0)$ & 0.006 \\
\hline Hypercholesterolemia & $115(26.7)$ & $54(23.4)$ & $61(30.5)$ & 0.095 \\
\hline
\end{tabular}

Data are presented as $n(\%)$ or mean \pm standard deviation

$B E V$ bevacizumab, $B M I$ body mass index, $C O P D$ chronic obstructive pulmonary disease, $C P$ carboplatin + paclitaxel, ECOG Eastern Cooperative Oncology Group, PS performance status, TIA transient ischemic attack

${ }^{a} p$ values $\leq 0.05$ were bolded and considered statistically significant

comorbidity index, was similar between cohorts. However, the cohorts differed significantly in the prevalence of anemia, history of serious bleeding events, weight loss, and venous thromboembolism, with the $\mathrm{Bev}+\mathrm{CP}$ cohort significantly less likely to have these conditions at baseline than the CP cohort.

Similar trends in patient characteristics noted in the overall population were found in each of the four subpopulations (data not shown). In general, the elderly population had more comorbidities than the total patient population. The subpopulation of 96 patients with brain/ CNS metastases was slightly younger than the total population (mean age 61 years) and was less likely to have comorbid conditions.

In the overall population, the $\mathrm{Bev}+\mathrm{CP}$ cohort had a higher average number of chemotherapy cycles (five vs. four), with a consequently higher proportion treated according to label, defined as receiving four or more cycles of therapy (72 vs. $50 \%$ ) than the CP cohort (Table 2). The most common reason for discontinuation of induction therapy in both the Bev $+\mathrm{CP}$ and the $\mathrm{CP}$ cohorts was disease progression, followed by toxicity.

Less than half of both cohorts received maintenance therapy after induction, with more than twice the proportion of $\mathrm{Bev}+\mathrm{CP}$ patients receiving maintenance therapy compared with CP patients: 45 vs. $21 \%$ (Table 2). Disease progression was the primary reason for not receiving maintenance therapy. For those receiving maintenance therapies, the majority of the $\mathrm{Bev}+\mathrm{CP}$ cohort received bevacizumab, whereas the majority of the $\mathrm{CP}$ cohort received pemetrexed and erlotinib. Second-line therapy was initiated in $54 \%$ of Bev $+\mathrm{CP}$ patients and in $44 \%$ of $\mathrm{CP}$ patients, with pemetrexed prescribed most frequently. These treatment patterns were similar for the other 
Table 2 Treatment patterns during follow-up in the overall population and elderly subpopulation

\begin{tabular}{|c|c|c|c|c|}
\hline & \multicolumn{2}{|l|}{ All patients } & \multicolumn{2}{|c|}{ Elderly subpopulation } \\
\hline & $\begin{array}{l}\mathrm{BEV}+\mathrm{CP} \\
(n=231)\end{array}$ & $\begin{array}{l}\text { CP } \\
(n=200)\end{array}$ & $\begin{array}{l}\mathrm{BEV}+\mathrm{CP} \\
(n=137)\end{array}$ & $\begin{array}{l}\mathrm{CP} \\
(n=94)\end{array}$ \\
\hline \multicolumn{5}{|l|}{ First-line induction therapy } \\
\hline Mean (median) number of cycles & $5(4.0)$ & $4(3.5)$ & $5(4.0)$ & $5(4.0)$ \\
\hline Patients treated according to label $\geq 4$ cycles & $166(71.9)$ & $100(50.0)$ & $98(71.5)$ & $49(52.1)$ \\
\hline Discontinued induction therapy ${ }^{a}$ & $116(50.2)$ & $117(58.5)$ & $63(46.0)$ & $58(61.7)$ \\
\hline \multicolumn{5}{|l|}{ Reasons for discontinuation } \\
\hline Disease progression & $61(52.6)$ & $72(61.5)$ & $25(39.7)$ & $31(53.4)$ \\
\hline Drug shortage & $0(0.0)$ & $1(0.9)$ & $0(0.0)$ & $1(1.7)$ \\
\hline Drug toxicity & $36(31.0)$ & $23(19.7)$ & $24(38.1)$ & $12(20.7)$ \\
\hline Patient request & $6(5.2)$ & $6(5.1)$ & $5(7.9)$ & $4(6.9)$ \\
\hline Patient on active surveillance & $0(0.0)$ & $2(1.7)$ & $0(0.0)$ & $2(3.4)$ \\
\hline Patient to receive surgery/radiation & $2(1.7)$ & $4(3.4)$ & $1(1.6)$ & $2(3.4)$ \\
\hline Patient too ill for further chemotherapy & $10(8.6)$ & $9(7.7)$ & $7(11.1)$ & $6(10.3)$ \\
\hline Patient on Coumadin & $1(0.9)$ & $0(0.0)$ & $1(1.6)$ & $0(0.0)$ \\
\hline \multicolumn{5}{|l|}{ Maintenance therapy } \\
\hline Received maintenance & $103(44.6)$ & $42(21.0)$ & $67(48.9)$ & $20(21.3)$ \\
\hline \multicolumn{5}{|c|}{$\begin{array}{l}\text { Reasons for no maintenance therapy after induction } \\
\text { (if mentioned) in those without maintenance }\end{array}$} \\
\hline Disease progression & $75(58.6)$ & $76(48.1)$ & $34(48.6)$ & $30(40.5)$ \\
\hline Patient on active surveillance & $20(15.6)$ & $48(30.4)$ & $12(17.1)$ & $24(32.4)$ \\
\hline Patient request & $4(3.1)$ & $3(1.9)$ & $4(5.7)$ & $3(4.1)$ \\
\hline Patient too ill for further chemotherapy & $10(7.8)$ & $20(12.7)$ & $7(10.0)$ & $11(14.9)$ \\
\hline Unknown & $19(14.8)$ & $11(7.0)$ & $13(18.6)$ & $6(8.1)$ \\
\hline Discontinued maintenance therapy & $93(90.3)$ & $33(78.6)$ & $59(88.1)$ & $15(75.0)$ \\
\hline \multicolumn{5}{|l|}{ Reasons for discontinuation } \\
\hline Disease progression & $74(79.6)$ & $27(81.8)$ & $45(76.3)$ & $11(73.3)$ \\
\hline Toxicity & $7(7.5)$ & $1(3.0)$ & $5(8.5)$ & $0(0.0)$ \\
\hline Patient request & $5(5.4)$ & $2(6.1)$ & $2(3.4)$ & $1(6.7)$ \\
\hline Patient on active surveillance & $5(5.4)$ & $1(3.0)$ & $5(8.5)$ & $1(6.7)$ \\
\hline Patient too ill for further chemotherapy & $1(1.1)$ & $2(6.1)$ & $1(1.7)$ & $2(13.3)$ \\
\hline Patient to receive surgery & $1(1.1)$ & $0(0.0)$ & $1(1.7)$ & $0(0.0)$ \\
\hline \multicolumn{5}{|c|}{ Maintenance agents ( $\%$ of those with maintenance) } \\
\hline Bevacizumab & $83(80.6)$ & $4(9.5)$ & $55(82.1)$ & $2(10.0)$ \\
\hline Carboplatin & $3(2.9)$ & $7(16.7)$ & $2(3.0)$ & $3(15.0)$ \\
\hline Paclitaxel & $0(0.0)$ & $3(7.1)$ & $0(0.0)$ & $1(5.0)$ \\
\hline Erlotinib & $10(9.7)$ & $9(21.4)$ & $5(7.5)$ & $4(20.0)$ \\
\hline Pemetrexed & $16(15.5)$ & $21(50.0)$ & $9(13.4)$ & $10(50.0)$ \\
\hline Docetaxel & $1(1.0)$ & $5(11.9)$ & $1(1.5)$ & $3(15.0)$ \\
\hline Gemcitabine & $4(3.9)$ & $3(7.1)$ & $3(4.5)$ & $1(5.0)$ \\
\hline Vinorelbine & $1(1.0)$ & $0(0.0)$ & $1(1.5)$ & $0(0.0)$ \\
\hline Other (cetuximab, cisplatin) & $2(1.9)$ & $2(4.8)$ & $1(1.5)$ & $1(5.0)$ \\
\hline \multicolumn{5}{|l|}{ Second-line therapy } \\
\hline Received second-line & $124(53.7)$ & $87(43.5)$ & $71(51.8)$ & $36(38.3)$ \\
\hline \multicolumn{5}{|l|}{ Second-line agents } \\
\hline Bevacizumab & $39(31.5)$ & $11(12.6)$ & $22(31.0)$ & $6(16.7)$ \\
\hline Carboplatin & $26(21.0)$ & $16(18.4)$ & $14(19.7)$ & $7(19.4)$ \\
\hline Paclitaxel & $8(6.5)$ & $6(6.9)$ & $6(8.5)$ & $1(2.8)$ \\
\hline Erlotinib & $31(25.0)$ & $15(17.2)$ & $21(29.6)$ & $9(25.0)$ \\
\hline
\end{tabular}


Table 2 continued

\begin{tabular}{|c|c|c|c|c|}
\hline & \multicolumn{2}{|l|}{ All patients } & \multicolumn{2}{|c|}{ Elderly subpopulation } \\
\hline & $\begin{array}{l}\mathrm{BEV}+\mathrm{CP} \\
(n=231)\end{array}$ & $\begin{array}{l}\mathrm{CP} \\
(n=200)\end{array}$ & $\begin{array}{l}\mathrm{BEV}+\mathrm{CP} \\
(n=137)\end{array}$ & $\begin{array}{l}\mathrm{CP} \\
(n=94)\end{array}$ \\
\hline Pemetrexed & $58(46.8)$ & $40(46.0)$ & $28(39.4)$ & $16(44.4)$ \\
\hline Docetaxel & $5(4.0)$ & $7(8.0)$ & $2(2.8)$ & $2(5.6)$ \\
\hline Gemcitabine & $16(12.9)$ & $15(17.2)$ & $9(12.7)$ & $4(11.1)$ \\
\hline Vinorelbine & $1(0.8)$ & $2(2.3)$ & $1(1.4)$ & $2(5.6)$ \\
\hline $\begin{array}{l}\text { Other (temozolomide, methotrexate, vinflunine, } \\
\text { clinical trial drug) }\end{array}$ & $8(6.5)$ & $12(13.8)$ & $4(5.6)$ & $4(11.1)$ \\
\hline
\end{tabular}

Data are presented as $n(\%)$

$B E V$ bevacizumab, $C P$ carboplatin + paclitaxel

${ }^{a}$ Represents those who discontinued prior to completion of all planned induction cycles

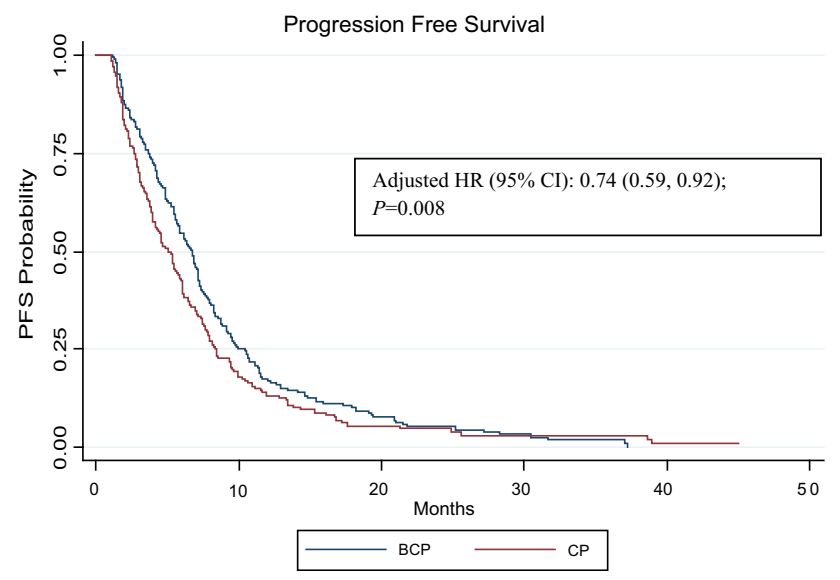

Fig. 2 Kaplan-Meier progression-free survival curve by treatment with vs without bevacizumab. $B$ bevacizumab, $C I$ confidence interval, $C P$ carboplatin + paclitaxel, $H R$ hazard ratio, $P F S$ progression-free survival

subpopulations (only data for elderly patients are shown in Table 2).

Bev + CP was associated with significantly longer median PFS for the full study population compared with the CP cohort (6.7 vs. 5.1 months; $p=0.041)$ (Fig. 2). After adjusting for covariates in the Cox regression model, $\mathrm{Bev}+\mathrm{CP}$ was associated with a $26 \%$ reduction in the risk of progression (HR 0.74; $95 \%$ CI $0.59-0.92$; $p=0.008$ ). Similar results for PFS were noted in the elderly and brain/ CNS metastases subpopulations but not in the ECOG PS score $\geq 2$ and anticoagulation subpopulations (Fig. 3). Specifically, in the elderly subpopulation, treatment with $\mathrm{Bev}+\mathrm{CP}$ was associated with a $35 \%$ reduction in the risk of progression, with a median PFS advantage of 1.8 months compared with the $\mathrm{CP}$ cohort. The brain/CNS metastases subpopulation had a $51 \%$ reduction in risk of progression, with a median PFS advantage of 2.1 months. Median PFS advantage was $\leq 1$ month in patients with an ECOG PS score $\geq 2$ and in the anticoagulation subpopulation.

Treatment with Bev + CP was associated with significantly longer median OS (by 2.9 months) over the $\mathrm{CP}$ cohort (11.9 vs. 9.0 months; $p<0.001)$ (Fig. 4). A significantly lower proportion of the $\mathrm{Bev}+\mathrm{CP}$ cohort died during follow-up compared with the CP cohort (72 vs. 80 $\%)$. After adjusting for covariates in the Cox PH model, Bev + CP treatment was associated with a $43 \%$ reduction
Fig. 3 Adjusted hazard ratios for progression-free and overall survival in study subpopulations. CNS central nervous system, ECOG Eastern Cooperative Oncology Group

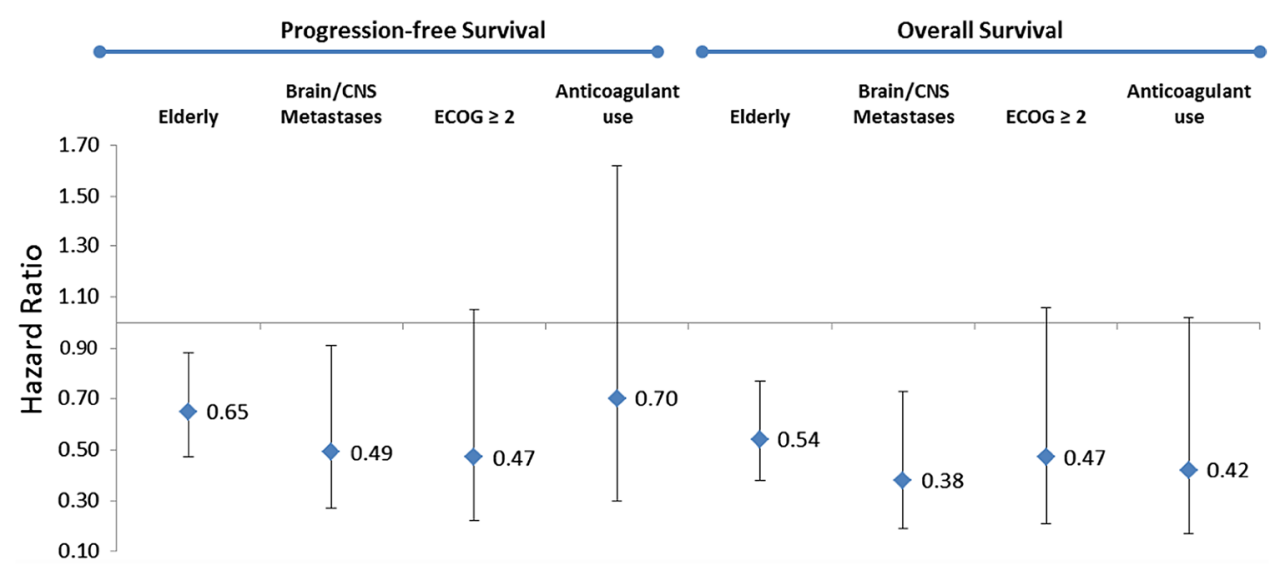




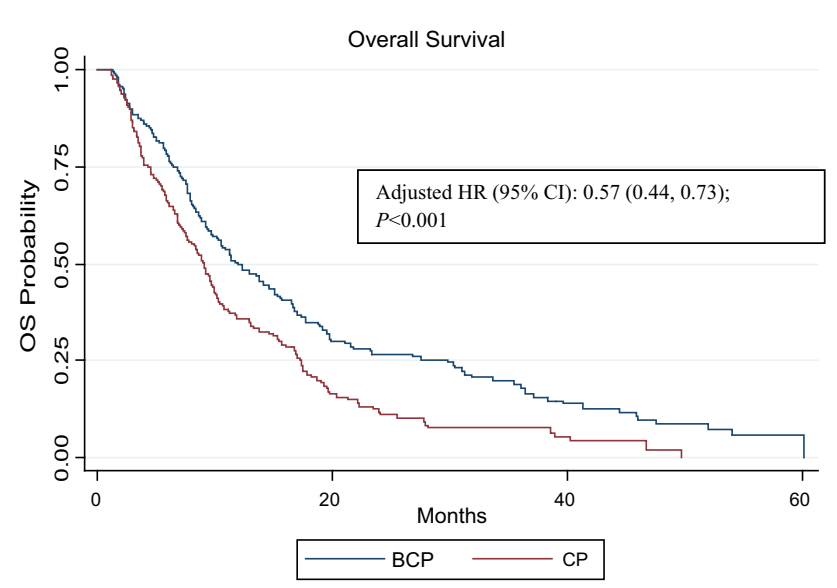

Fig. 4 Kaplan-Meier overall survival curve by treatment with vs without bevacizumab. $B$ bevacizumab, $C I$ confidence interval, $C P$ carboplatin + paclitaxel, $H R$ hazard ratio, $O S$ overall survival

in the risk of death (HR $0.57 ; 95 \%$ CI $0.44-0.73 ; p<$ 0.001). Similarly, an OS advantage was found in the elderly and brain/CNS metastases subpopulations, with significant adjusted risk reductions of $46 \%$ (median OS 12.3 vs. 8.3 months, respectively; $p=0.001$ ) and $62 \%$ (median OS 11.3 vs. 9 months, respectively; $p=0.010$ ) (Fig. 3). Similar to PFS, a trend for longer survival was noted in the Bev $+\mathrm{CP}$ cohort in patients with an ECOG PS $\geq 2$ and in the anticoagulation subpopulations, but the difference was not statistically significant.

\section{Discussion}

The present study provides real-world evidence of the effectiveness of using bevacizumab in combination with carboplatin and paclitaxel as first-line therapy for advanced/metastatic NS-NSCLC. Treatment with Bev + $\mathrm{CP}$ was associated with a significantly longer median PFS by $\sim 2$ months and median OS by 3 months compared with treatment with CP alone. Furthermore, in subset analyses, there remained a statistically significant improvement in median PFS and OS for patients treated with bevacizumab who were aged $\geq 65$ years and for those who had brain/ CNS metastases at initiation of therapy. Although sufficient evidence is available from clinical trials of the efficacy of bevacizumab, there is limited evidence for key subpopulations because of clinical trials excluding or under-representing vulnerable patient populations. The present study included these vulnerable populations and suggests that bevacizumab should be considered as first-line therapy in advanced/metastatic NS-NSCLC in these patient populations.

Specifically, in the elderly subpopulation of NS-NSCLC patients, evidence has been conflicting. In the phase III ECOG 4599 trial, which reported a median OS advantage of 2 months with the addition of bevacizumab to $\mathrm{CP}$, subgroup analysis could not confirm that this advantage was seen in patients aged $\geq 65$ years [7]. In addition, a pooled analysis of two phase III trials, ECOG 4599 and PointBreak, found that the addition of bevacizumab to chemotherapy backbone (CP in ECOG 4599 and carboplatin/pemetrexed in PointBreak) was associated with a significant reduction in the risk of death for patients aged $<75$ years; however, the analysis could not confirm this benefit for the 157 patients across these two studies who were aged $\geq 75$ years [12]. A number of real-world evaluations have since attempted to further define the role of bevacizumab in this population. The ARIES trial was a prospective multicenter observational cohort study that enrolled 1967 patients with advanced or metastatic NSCLC who were receiving bevacizumab in combination with firstline chemotherapy [13]. After adjusting for potential differences in patient characteristics, the risk of progression for older patients was similar to that for younger patients (HR 1.01; $95 \%$ CI $0.92-1.10$ for patients aged $\geq 65$ vs. aged $<65$ years), but the risk of death was higher (HR 1.17; $95 \%$ CI $1.06-1.28$ for patients aged $\geq 65$ vs. aged $<65$ years); however, given that all patients in this analysis received bevacizumab, this study was not designed to assess the benefit of bevacizumab added to chemotherapy over chemotherapy alone for either group [13]. Langer et al. [14] examined the addition of bevacizumab to chemotherapy in a Medicare population of patients with NS-NSCLC who were aged $\geq 65$ years and found that bevacizumab imparted a statistically significant benefit in terms of OS for patients aged $\geq 65$ years and for those aged $\geq 75$ years. However, Zhu et al. [15] conducted a large population-based study using SEER data for Medicare beneficiaries and could not confirm the effectiveness of bevacizumab added to $\mathrm{CP}$ in the elderly subpopulation in terms of survival advantage. In our study, in the elderly subpopulation of 231 patients, treatment with Bev $+\mathrm{CP}$ was associated with a 4-month survival advantage versus those who did not receive $\mathrm{Bev}+\mathrm{CP}$. These findings are consistent with both the meta-analysis of ECOG 4599 and PointBreak and the real-world analysis by Langer and colleagues $[12,14]$. The discordance observed by the latter analysis of Medicare patients may be explained by the changing treatment patterns during the time frame in which Zhu et al. [15] conducted their study versus Langer et al. [14] and our analysis, resulting in an increased patient selection in the elderly population receiving bevacizumab. Zhu et al. [15] identified patients initiating bevacizumab in 2006 and 2007 (the first 2 years after approval of bevacizumab in the USA); of the 1502 patients treated with Bev $\pm \mathrm{CP}$, only $318(21 \%)$ received bevacizumab, and a greater proportion of the bevacizumab arm had stage IV disease (82.4 vs. $70.9 \% ; p \leq 0.001)$. In contrast, Langer 
et al. [14] included a 4-year follow-up after the approval of bevacizumab (2006-2009) and found that $35 \%$ of the 1706 patients enrolled received bevacizumab; 74.2 and $73.6 \%$ of those treated with Bev $+\mathrm{CP}$ and CP alone, respectively, had stage IV disease.

Further, our study evaluated data from 2006 to 2013, and key changes in treatment patterns also occurred during this time frame that may have contributed to the survival advantage of the Bev $+\mathrm{CP}$ cohort in the elderly subpopulation. During this time, Weiss et al. [16] examined second-line therapy in the elderly population; they found it to have the same benefit as in their younger counterparts. In our study, $51.8 \%$ of patients aged $\geq 65$ years went on to receive second-line therapy in the Bev + $\mathrm{CP}$ arm versus $38.3 \%$ in the $\mathrm{CP}$ arm; pemetrexed was the predominant therapy in this line. This is numerically similar to that reported in the ECOG 4599 trial for the bevacizumab cohort for the entire population (51\%), although in the cohort of patients aged $\geq 75$ years, more patients in the non-bevacizumab cohort received postprogression therapy (52 vs. $40 \%$ ) [12]. While Zhu et al. [15] did not report these data, based on the timing of study enrollment this may also have contributed to the difference in OS benefit seen in our population versus that study.

Clinicians have been reluctant to use bevacizumab in patients with brain/CNS metastases because of the risk of hemorrhage. This imposes a real limit to the widespread use of bevacizumab, as 25-30\% of patients with NSCLC will ultimately be diagnosed with brain metastases, and this is also often the first site of recurrence in patients initially treated for early-stage disease [17]. Besse et al. [18] reviewed clinical trial data to assess the risk of cerebral hemorrhage in patients with CNS metastases treated with bevacizumab for various solid tumors. Data from 187 (bevacizumab-treated, $n=91$; non-bevacizumab-treated, $n=96$ ) patients were extracted from 13 randomized controlled trials of patients who had undiagnosed CNS metastases at therapy initiation or developed CNS metastasis during treatment and hence were evaluable for a safety analysis [18]. The rate of cerebral hemorrhage in the bevacizumab-treated group was 3.3 vs. $1.0 \%$ in those not treated with bevacizumab [18]. In addition, this study also analyzed two additional datasets (one obtained from two single-arm, open-label safety studies that also excluded CNS metastases at diagnosis and one obtained from two studies that allowed patients with treated CNS metastases to be included); 321 and 131 bevacizumab-treated patients with CNS metastases from these two datasets, respectively, were analyzed, with the rates of cerebral hemorrhage reported at 0.9 and $0.8 \%$, respectively [18]. An observational cohort study, the ARIES trial, enrolled NSCLC patients treated with bevacizumab in combination with chemotherapy who were not represented in the larger randomized controlled trials and found no reports of grade 3 or higher CNS bleeds in the 150 patients with CNS metastases at baseline [19]. Additionally, a small phase II trial [20] evaluated the efficacy and safety of bevacizumab combination therapy in patients with NS-NSCLC and asymptomatic untreated brain metastases. In the 67 patients treated with first-line Bev $+\mathrm{CP}$, the median OS was 16.0 months (95\% CI 12-21), and no CNS bleeds were reported [20]. In our study, treatment with Bev + CP was associated with a statistically significant increase of 2 months in median OS versus treatment with CP. These data seem to suggest that the presence of brain/CNS metastases should not necessarily exclude patients from treatment with bevacizumab.

As with all observational research, there are limitations that must be considered to allow for valid interpretation of the study findings. The key limitation is that of treatment selection bias, with the relative contraindications for bevacizumab (bleeding, brain metastases) increasing the likelihood of patients with these conditions receiving $\mathrm{CP}$. Additionally, specifically with the elderly patients, physicians are likely to select the healthiest patients for aggressive systemic treatment with bevacizumab. While the study did adjust for these differences in the Cox $\mathrm{PH}$ models and also excluded patients who died or progressed within the first 30 days, the nonrandomized nature of the study does not likely fully account for the inherent treatment selection bias. Additionally, concern regarding the tolerability of a doublet platinum therapy in patient populations with an ECOG PS $\geq 2$ may have contributed to the rather small sample size for this subset. While data were collected through a multimodal approach, it is still possible that important data elements remained unavailable. For example, some of the variables used as patient selection criteria (e.g., non-squamous histology) may have been missing from the data source, which would have resulted in the exclusion of patients who would have otherwise been eligible, or variables used to describe the eligible patient population and variables used to classify patients into subpopulations (e.g., ECOG PS) were not available for all patients. Additionally, any healthcare utilization outside of the oncology practices reporting to ION was not captured for these patients (e.g., drugs administered or imaging performed during hospitalizations, emergency department visits, and primary care or non-oncology specialist visits), and therefore indicators of progression or change in therapy may not have been captured. However, while data availability may have imposed some limitations on the results, it is unlikely that the cohorts were differentially biased by these data limitations. 


\section{Conclusion}

Based on this retrospective analysis, the addition of bevacizumab to $\mathrm{CP}$ is an effective treatment strategy for patients with NS-NSCLC in terms of PFS and OS improvement. Included in this analysis were patients traditionally excluded from bevacizumab therapy, and subset analysis reveals that those patients with brain/CNS metastases or aged $\geq 65$ years maintain a statistically significant benefit in terms of OS and PFS with the addition of bevacizumab.

Acknowledgments The authors would like to acknowledge Marlo Blazer for her medical writing services.

\section{Compliance with Ethical Standards}

Funding Funding for the research study and resultant publication was provided by Genentech, Inc.

Conflict of interest HPG and AR are employees of Genentech and own company stock. SJH was an employee of Genentech at the time of this study and during manuscript preparation. OEL, ADC, MRG, $\mathrm{JW}$, and ME are employees of Xcenda, which has received research funding from Genentech for the conduct of this study and for the preparation of this manuscript.

Ethical approval Formal consent is not required for this type of study. This article does not contain any studies with animals performed by any of the authors.

Open Access This article is distributed under the terms of the Creative Commons Attribution-NonCommercial 4.0 International License (http://creativecommons.org/licenses/by-nc/4.0/), which permits any noncommercial use, distribution, and reproduction in any medium, provided you give appropriate credit to the original author(s) and the source, provide a link to the Creative Commons license, and indicate if changes were made.

\section{References}

1. Seigel RL, Miller KD, Jemal A. Cancer statistics 2015. CA Cancer J Clin. 2015;65:5-29.

2. Molina JR, Yang P, Cassivi SD. Non-small cell lung cancer: epidemiology, risk factors, treatment, and survivorship. Mayo Clin Proc. 2008;83(5):584-94.

3. Schiller JH, Harrington D, Belani CP, et al. Comparison of four chemotherapy regimens for advanced non-small-cell lung cancer. N Engl J Med. 2002;346:92-8.

4. Socinski MA, Schell MJ, Peterman A, et al. Phase III trial comparing a defined duration of therapy versus continuous therapy followed by second-line therapy in advanced-stage IIIB/IV non-small-cell lung cancer. J Clin Oncol. 2002;20:1335-43.

5. Sandler A, Yi J, Dahlberg S, et al. Treatment outcomes by tumor histology in Eastern Cooperative Group Study E4599 of Bevacizumab with paclitaxel/carboplatin for advanced non-small cell lung cancer. J Thorac Oncol. 2010;5:1416-23.
6. Scagliotti GV, Parikh P, von Pawel J, et al. Phase III study comparing cisplatin plus gemcitabine with cisplatin plus pemetrexed in chemotherapy-naïve patients with advanced-stage nonsmall-cell lung cancer. J Clin Oncol. 2008;26:3543-51.

7. Sandler A, Gray R, Perry MC, et al. Paclitaxel-carboplatin alone or with bevacizumab for non-small-cell lung cancer. $\mathrm{N}$ Engl $\mathrm{J}$ Med. 2006;355:2542-50.

8. Reck M, von Pawel J, Zatloukal P, et al. Phase III trial of cisplatin plus gemcitabine with either placebo or bevacizumab as first-line therapy for nonsquamous non-small-cell lung cancer: AVAiL. J Clin Oncol. 2009;27:1227-34.

9. National Comprehensive Cancer Network. NCCN Practice Guidelines in Oncology-v.7.2015: non-small cell lung cancer. Available at: http://www.nccn.org/professionals/physician_gls/ pdf/nscl.pdf. Accessed Aug 122015.

10. Leighl NB, Bennouna J, Yi J, et al. Bleeding events in bevacizumab-treated cancer patients who received full-dose anticoagulation and remained on study. Br J Cancer. 2011;104(3): 413-8.

11. Johnson DH, Fehrenbacher L, Novotny WF, et al. Randomized phase II trial comparing bevacizumab plus carboplatin and paclitaxel with carboplatin and paclitaxel alone in previously untreated locally advanced or metastatic non-small-cell lung cancer. J Clin Oncol. 2004;22(11):2184-91.

12. Langer CJ, Socinski MA, Patel JD, et al. Isolating the role of bevacizumab in elderly patients with previously untreated nonsquamous non-small cell lung cancer: secondary analyses of the ECOG 4599 and PointBreak trials. Am J Clin Oncol. doi:10. 1097/COC.0000000000000163.

13. Wozniak AJ, Kosty MP, Jahanzeb M, et al. Clinical outcomes in elderly patients with advanced non-small cell lung cancer: results from ARIES, a bevacizumab observational cohort study. Clin Oncol. 2015;27:187-96.

14. Langer C, Ravelo A, Hazard SJ, et al. Comparison of survival and hospitalization rates between Medicare patients with advanced NSCLC treated with bevacizumab-carboplatin-paclitaxel and carboplatin-paclitaxel: a retrospective cohort study. Lung Cancer. 2014;86:350-7.

15. Zhu J, Sharma DB, Gray SW, et al. Carboplatin and paclitaxel with vs without bevacizumab in older patients with advanced non-small cell lung cancer. JAMA. 2012;307(15):1593-601.

16. Weiss GJ, Langer C, Rosell R, et al. Elderly patients benefit from second-line cytotoxic chemotherapy: subset analysis of a randomized phase III trial of pemetrexed compared to docetaxel in patients with previously treated advanced non-small-cell lung cancer. J Clin Oncol. 2006;24(27):4405-11.

17. Schettino C, Bareschino MA, Rossi A, et al. Targeting angiogenesis for treatment of NSCLC brain metastases. Curr Cancer Drug Targ. 2012;12:289-99.

18. Besse B, Lasserre SF, Compton P, Huang J, Augustus S, Rohr UP. Bevacizumab safety in patients with central nervous system metastases. Clin Cancer Res. 2010;16(1):269-78.

19. Wozniak AJ, Garst J, Jahanzeb M, et al. Clinical outcomes (CO) for special populations of patients (pts) with advanced non-small cell lung cancer (NSCLC): results from ARIES, a bevacizumab (BV) observational cohort study (OCS). J Clin Oncol. 2010;28:15s (suppl;abstr7618).

20. Besse B, Le Moulec S, Maziéres J, et al. Bevacizumab in patients with nonsquamous non-small cell lung cancer and asymptomatic, untreated brain metastases (BRAIN): a nonrandomized, phase II study. Clin Cancer Res. 2015;21(8):1896-903. 